\title{
Automatic Detection of Weld Defects Based on Hough Transform
}

\author{
Chiraz AJMI ${ }^{12}$, Sabra El FERCHICHI ${ }^{2}$, Abderrahmen ZAAFOURI ${ }^{1}$, Kaouther LAABIDI $^{12}$, \\ ${ }^{1}$ University of Tunis, National Superior Engineering School of Tunis, Street Taha Hussein Monfleury, 1008 Tunisia. \\ ${ }^{2}$ University Tunis El Manar, National Engineering School of Tunis, Analysis, Design and Control of Systems Laboratory \\ (LR11ES20), 1002, Tunisia
}

chiraz.ajmi@hotmail.fr sabra.elferchichi@enit.rnu.tn

abderrahmen.zaafouri@yahoo.fr Komri@uj.edu.sa

\begin{abstract}
Weld defect detection is an important application in the field of Non-Destructive Testing (NDT). These defects are mainly due to manufacturing errors or welding processes. In this context, image processing especially segmentation is proposed to detect and localize efficiently different types of defects. It is a challenging task since radiographic images have deficient contrast, poor quality and uneven illumination caused by the inspection techniques. The usual segmentation technique uses a region of interest $\mathrm{ROI}$ from the original image. In this article, a robust and automatic method is presented to detect linear defect from the original image without selection of ROI based on canny detector and a modified 'Hough Transform' technique. This task can be subdivided into the following steps: firstly, preprocessing step with Gaussian filter and contrast stretching; secondly, segmentation technique is used to isolate weld region from background and non-weld using Adaptative Thresholding and to extract edges; thirdly, detection, location of linear defect and limiting the welding area by Hough Transform. The experimental results show that our proposed method gives good performance for industrial radiographic images.
\end{abstract}

Keywords-Weld defect, radiography, NDT, Hough Transform, Canny, Adaptative Thresholding

\section{INTRODUCTION}

Radiography is one of the most used techniques in weld defect inspection. It could become a challenging task since radiographic films usually have noise and deficient contrast due to intrinsic factors involved in the inspection technique, such as non-uniform illumination and the limited range of intensities of the image capture device [1].

The traditional interpretation of radiographic images by artificial methods is subjective, time-consuming, and easy to cause fatigue, due to the problems associated with manual detection. In order to improve the automation level, there is currently a great deal of work and research on non- destructive testing (NDT) methods for detecting welding defects $[31,25$, 26].

Generally, there are five major types of weld defects: incomplete fusion, incomplete penetration, slag inclusion, gas cavity and crack which are summarized in Fig.1.

The research goal is to develop an automated method using image processing detection of defects techniques that is precise and objective. The purpose of the analysis process's automation is to reduce the analysis time, eliminate the subjective aspect in the analysis done by the inspectorand improve the quality of those images.

However, Image segmentation is a fundamental topic in image processing and it is often considered the most complex task in the processing of images. Researches in this area are copious but specific to the material being analyzed. In the case of the present investigation, different strategies and methods have been evaluated in term of defects detection in welding images $[9,10]$. The literature reviewed includes a large quantity and variety of methodologies for the detection of welding defects, such as interpolating the image's background curves, neuronal networks for classification but the inconvenient of this technique resides in its speed of execution that is slow enough $[7,8]$.

However, several works are made for segmentation such as Thresholding techniques which have been studied extensively. Large number of Thresholding methods [2, 27] have been published such as in [3] authors applied sauvola Thresholding with mathematical morphology for weld seam extraction and the Geometric Features for classification. Moreover, some researchers have used system of pattern recognition based on texture features analysis [4].

The results are impressive, but require a complex implementation and significant computing time. Furthermore, several researchers of active contours (snakes, level set) [28] have been often applied to deal with contour estimation of weld defect problems, and they fail in the presence of noise. Moreover, they are sensitive to initialization.

In this work we focus on radiographic image of lengthened, smooth and rectangular shape called lack of penetration defect that occurs in weld is taken for this work.

The most pertinent segmentation techniques apply the procedure that selects the region of interest ROI of the defect from the original image but in this work, we will use all the image without selection of any specific region therefore automate the detection of line defect. So, the preoccupation of this work is to present a whole and automatic procedure of line defect detection: we don't have to use a ROI or interfere the expert to have a diagnosis. Because of the corruption aspect of our images with noise and the meeting of gray level, the segmentation step needs an effective procedure for region separation based on Thresholding method.

In this context, we propose to use Adaptative thresholding, canny detector to extract edges and Hough transform to effectively segment the line weld defect in our images 
In fact, the standard Hough Transform is an efficient technique to find and link line segments in an image $[5,6,12,23]$.

Indeed, it is a transform between image space and parameter space, and it is suitable to detect a particular shape within an image. It is used to detect lines in pipe (crack), cable inspection, tracking road sign identification, lane detection, and many other industrial and medical applications. Moreover, it treats the cases of noisy shapes due to its voting scheme and it can be extended to detect shapes other than lines [26].

At First, a preprocessing stage for edge detection is made to differentiate between real intensity transitions and sudden noise transitions which is an important problem and each algorithm treats it differently $[25,4]$. Secondly, the Adaptative Thresholding which gives a threshold for each pixel in the image, to better recognize region and to avoid elimination of the same defect. Furthermore, edge extraction by canny is prior to reduce the amount of data in an image, while preserving the structural properties to be used for defect recognition and location.

This article is organized as follows: Section 1 presents an overview of related work in this context. In section 2, we will detail the proposed method for line defect and the solution scheme of the segmentation procedure developed in this study. Section 3 presents image pre-processing to ameliorate the quality of the image. Section 4 presents the obtained results.

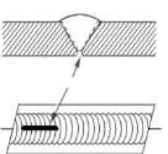

a.

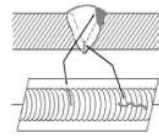

b.

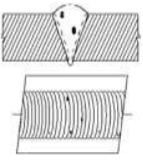

c.

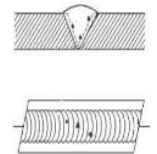

d.
Fig. 1. Weld defects. (a) crack; (b) lack of penetration, (c) porosity, (d) slag inclusion.

\section{RELATED WORK}

Many related work for linear shape detection has been carried out in recent years in many domains. Hough Transfrom (HT) has been regarded as a robust detection approach for analytic shapes such as lines, ellipses as well as arbitrary shapes [12, 13]. It is widely used in the detection of straight lines [24].

In [29], an approach is presented for using the HT in detecting line segments. A set of small windows are used to divide the task of finding line segments in an image into a set of subtasks of detecting short line segments in small windows. After obtaining line segments from windows, a combining strategy is used to combine collinear and overlapping line segments into one line segment.

Researches in [30] find and track road lanes using lines snakes. The Hough transform is used to obtain an initial position estimation of the lane boundaries on the road. Then the line snake improves the initial approximation to an accurate configuration of the lane boundaries. In [23] propose an new approach based on texture segmentation and on a variation of the Standard Hough Transform, in which the choice of the parameters that determine the straight line that better represents the image is based on the contour conditions of the particular case of weld line detection on fuel storage tanks.

In [5] detection follows a pattern recognition methodology to extract features and exploit SVM technique to classify the features. Actually, in every block that contains the defect, they perform Hough transform to remove the noisy pixels (removing pixels which the distance to detected line is larger than a fixed threshold) and get the accurate segmentation and location of the defect.

The authors in [6] use defect tracking in real-time radiographic image sequence of a moving weld. A defect segmentation algorithm with low threshold is used to segment all of the potential weld defects in each image of the sequence. Then the modified Hough transform is employed to track the center of gravity of potential defects in image sequence. The potential defects that cannot be tracked are eliminated as false contours.

In [22] authors propose an active contour model-based intimamedia segmentation approach. It simultaneously estimates the initial contour by using Hough transform on partitioned image segments. They combine the independent deformations of two contours together by minimizing a unified energy functional.

\section{PROPOSED METHOD FOR LINEAR WELD DEFECT RECOGNITION}

To remedy the problem of linear defect detection and localization in noisy condition image. We propose a four stages approach based on Hough transform. Each stage consists of a number of processes, which are carried out sequentially in order to correctly interface with the input of the following stage until the recognition report is obtained.

It is often necessary to start with the preprocessing stage; digital image processing techniques are employed to lessen the noise effects using Gaussian filter and to improve the contrast applying contrast stretching method. So that the principal objects in the image become more apparent than the background. In addition, segmentation stage: using an Adaptative segmentation method for region separation and edge detection. Canny catch as many edges as possible but it is accompanied with discontinuous and fine contours.

So, we applied local Thresholding and the binarization before canny to better separate the defect zone from background and to minimize the false contours and to avoid the elimination of same defect. Finally, the limitation of weld's region from the binarized image and the determination of shapes using Hough Transform is conducted. Hough linear Transform gives a meaningful set of lines that are weld and no-weld.

Anyway, the variation of Hough's parameters is done to find lines that are weld and the external lines which limits the defect. The flowchart of the proposed approach is shown in Fig. 2. The functionality of each stage will be described in more detail in the following sections. 


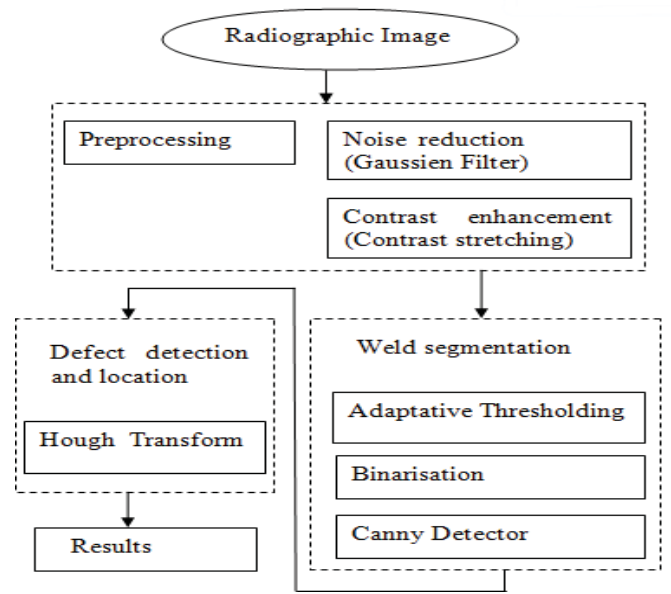

Fig. 2 Procedure for the automatic welding defect detection system. A. The pre- processing step

The film digitization process may produce small-contrast images containing some granularities. Image enhancement [15] is required to improve the visibility of the images by eliminating unwanted deformations or by ameliorating the contrast between the image background and the weld defect areas also for removing any noise resulting from the digitization process. Two original images representing lack of penetration defect (linear defect) are represented in Fig.3. In this section, the radiographic images are enhanced and filtered as described in the following sub-sections.

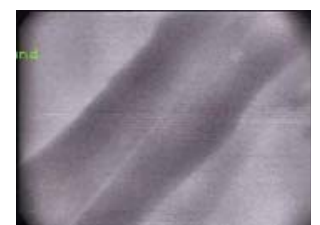

a.

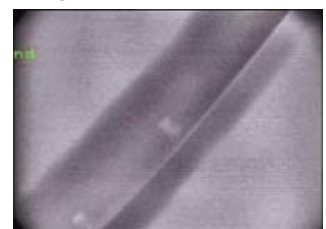

b.
Fig. 3. The original images. (a) Mono weld defect; (b) Multi weld defect.

1) Noise Reduction

The automatic acquisition of internal welding images is carried out via a linear X-ray sensor for weld inspection. These images obtained in the X-ray or gamma-ray imaging systems contain random fluctuations due to the statistics of the absorption of the quantum of radiation whereas the fundamental noise is due to the discrete nature of the X-ray photons $[16,17]$. This noise can impede the reliability of the detection of small discontinuities. In Digitized film-screen images, other sources due to the digitizer, the structure of the intensifying fluorescent screen and the granularity of the film emulsion contribute to noise. So, a periodic noise was added by the system to the "ideal"image.

It is delicate to choose a standard filter for noise elimination [14]. The choice of an operator is not simple and quite often a compromise must be found between speed of execution, and quality of result. Thereby, the right choice must keep the improvement of the visibility of our images according to the characteristics of the noise. Compared to different filter, we have found in previous work [32] that gaussian filter was the appropriate one to apply to our images. It eliminates most of the noise and maintain clearer contours [18].

\section{2) Contrast enhancement}

It can sharpen the image border and improve the accuracy by accentuating the brightness difference between background and foreground. The idea behind enhancement techniques is to bring out detail that is obscured, or simply to highlight certain features of interest in an image [19].

In our work, we have applied the Adjust Contrast tool in MATLAB version which is an interactive contrast and brightness adjustment tool.

\section{B. Segmentation step}

The segmentation aims at extracting sets of points corresponding to significant parts of the "global shape" represented by the image [21].

In pixel detection we have Thresholding which is a widespread technique as it allows the conversions of an image in grey scale to a binary image in such a way as to separate background objects according to a specified threshold. The Watershed transform is used in region detection. This technique makes use of morphological mathematics and allows the generation of regions based on cavity filling by simulating a valley filling with water; in the measure that the water level raises, adjacent regions start forming unions.

Liao and $\mathrm{Ni}$ [25] proposed a method based on the observation that the intensities of pixels in the weld area distribute more as a Gaussian distribution than other areas in the image. This method had been proved effective, however, only to segment linear welds. Wang et al. [26] have combined image processing techniques: background and histogram Thresholding were implemented to separate defects from the background.

What it makes delicate the segmentation is the specification of our image. It has a gray levels concentration in a narrow band of values even using an optimal method for the choice of a global threshold. So, we have to calculate a threshold for each small region of similar pixel value in the image, to better recognize defect's zone. Precisely, the selection of local threshold to statistically examine the intensity values of the neighborhood of each pixel by using an adaptive segmentation algorithm. Thus, it can be useful for edge detection step without suppression of defects and good separation of regions.

Anyway, edges define the boundaries between regions in an image, which helps with segmentation and object recognition.

\section{1) Adaptative Thresholding and binarization}

Intensity is a distinguishing feature that can be used to localize the defects from the background. Thus, it is useful to have it preceded with segmentation step using thresholding for background subtraction. In fact, a global threshold would not be a good choice, we choose to apply an Adaptative Thresholding to separate desirable foreground image objects from the background based on the difference in pixel 
intensities of each region. The Local threshold is found by examining statistically (mean, median) the intensity values of the local neighborhood of each pixel Simple and fast functions include the mean of the local intensity distribution. Generally, the statistic chosen (mean) which is most appropriate depends largely on the input image.

This procedure also offers the possibility of using median filtering as a robust alternative to the mean. If the pixel value is below the threshold it is set to the background value, otherwise it assumes the foreground value. After adopting the value of threshold we use binarization to convert our image to a binary image.

\section{2) Canny Detector Step}

Canny edge detector $[11,20]$ approximates the operator that optimizes the localization. It is a good detector of edges for noisy images especially while combined to thresholding method. It improves the signal to noise-ratio and this is established by Non maxima suppression method. By fixing the values of Sensitivity threshold and the Standard deviation of the filter to 0.1 and 2. Finally, we can apply canny detector to detect the contours from binary image.

C. Line detection Step

1) Hough Transform method

Many researchers used Hough Transform for line detection $[22,23,5]$. Since it is a robust method that converts the straight line detection problem to a peak seeking problem in the parameter space (also called HT space or Hough space). Generally, a straight line is represented by slope and intercept of $y$-axis as follows:

$$
\mathrm{b}=\mathrm{y}-\mathrm{kx}
$$

Where $\mathrm{k}$ is the slope of the straight line, and $b$ is the intercept of the straight line with $y$-axis.

In the $(k, b)$ parameter space, a single point $(k, b)$ corresponds to a unique straight line in image space. This method for the detection of straight lines suffers from horizontal and vertical line detection problems because the slope and the intercept are unbounded. So it was suggested to replace the slope-intercept parameters by angle-radius parameters. The polar representation of straight lines is used as follows:

$$
\rho=x \cos \theta+y \sin \theta
$$

Where $\rho$ is the distance between the origin of the coordinate and the straight line 1 , and $\theta$ is the angle between $\rho$ and $X-$ axis. $\rho, \theta$ and 1 are shown in Fig. 4.

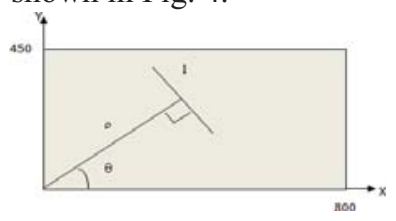

Fig. 4. Line detection

In the $(\rho, \theta)$ parameter space, a single point $(\rho, \theta)$ corresponds to a unique straight line. In practical implementations of line detection procedures, both $\theta$ and $\rho$ have to be quantized into several discrete values and each point $(\mathrm{x}, \mathrm{y})$ in the image has its relevant $(\rho, \theta)$. Then we can construct two dimensional accumulator array $\mathrm{H}(\rho, \theta)$ that denotes the number of the collinear points with respect to $(\rho, \theta)$ in the binary image. Last, the maximal $\mathrm{H}(\rho, \theta)$ is the peaks. In this way, the problem of detecting collinear points in image space is converted to the problem of finding peaks in parameter space. So, the line with the highest value of peak indicates the straight line of defect in the weld or external lines of defect zone.

\section{2) Segmenting the defect}

Canny gives the binary image with discrete point at the edges and where the intensity level changes. So these may or may not be in the same line. To detect the co-linearity of the points the Hough transform is then applied.

The contour extraction of the binarized image is performed to reduce the number of pixels to be processed by Hough transform. By observing Fig. 7 (a) and (c) we note that the distribution of defects is in the form of a line and our image contain more than one straight line which contains the defect and external lines which limits the defect.

By applying Hough, for segmented image resulting from canny and Thresholding procedure we aim to detect lines and recognize the defect's line. As shown in Fig. 4, we establish a XOY coordinate system with the origin at the left-bottom of the binary image. $\theta$ should be in the range $[-90,89)$, so that every pixel with value of 1 in the binary image can be detected. According to fig 4. and the dimension of the image we have the diagonal $\mathrm{OA}$ is the maximum value of the $\rho$ which is calculated as below :

$$
\rho<|\mathrm{OA}|=\sqrt{450^{2}+800^{2}=917}
$$

Therefore, the range of $\rho$ is $[0,917]$.

Consequently, we can get a two-dimensional accumulator array $H(\rho, \theta)$ with the size of $1835 \mathrm{X} 180$. The selected intervals of $\theta$ and $\rho$ are proved to meet the accuracy requirement for detection.

We calculate the values of the accumulator array $H(\rho, \theta)$ for all pixels with the value of 1 in the binary image. The corresponding parameter matrix of edge image and the sinusoidal lines are points in $\mathrm{X}-\mathrm{Y}$ plan and peaks point of Hough of Fig (3.b) are presented in Fig. 5. By taking $(\rho, \theta)$ corresponding to the maximal value of the accumulator array as the parameter of the most significant detecting line in the binary image we find that doesn't correspond to the defect's line. So, it is suitable to apply different post processing algorithms to detect the defect's line from others.

Indeed, we propose an algorithm to extract the defect's line by using the parameter of Hough $\rho$. The defect is always in the middle and is surrounded by two external lines so our goal is to detect and to draw the external lines and after that select the interior line which is the defect.

We note that those lines correspond to the two lines with min and the max of $\rho$. So we follow those steps:

-We have eliminated the lines corresponding to the borders of the image; 
-We consider that the line with a minimum value of $\rho$ is the first external line detected in the same as shown in Fig.8;

-We consider the first line with $\rho$ superior to the minimum and inferior to the maximum is our defect.

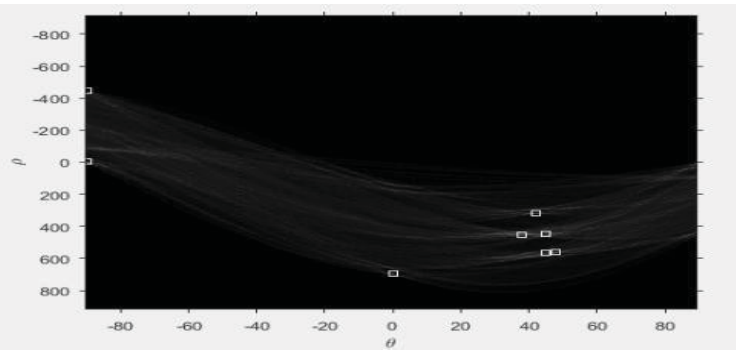

Fig. 5. Peak points in the Hough transform

\section{EXPERIMENTAL RESULTS AND DISCUSSIONS}

To validate the efficiency and robustness of our algorithm using our images. We applied our procedure already stated on our images. The performance of the proposed approach in noisy environments is detailed below.

\section{A. Preprocessing Results}

In Fig. 6, we can notice the effect of the image enhancement stage on two of the digitized radiographic images. The original, the enhanced image after Gaussian filter and contrast stretching effects of mono and multiple weld defects is shown in Fig. 6 (a) and (b) respectively. For quantitative evaluation of the image enhancement process, Peak Signal-to-Noise Ratio (PSNR) was applied before and after each process and original image is summarized in Table. I

TABLE. I. THE VALUE OF PSNR OF FIG (3. b)

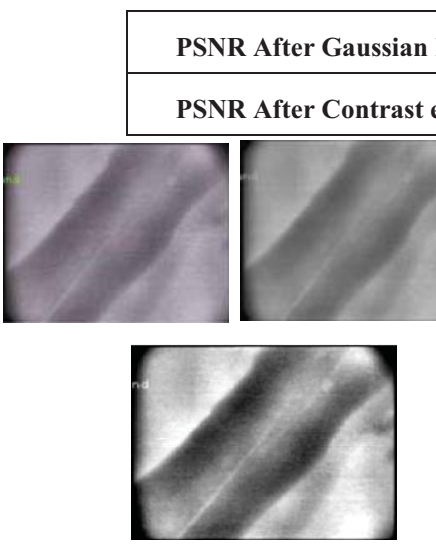

a.
Fig.6.Original image, Results of noise - removal and contrast enhancement by stretching (a) Applied to Fig (3.a); (b) Applied to Fig (3.b).

As we can see from the above figures, the implementation results of Gaussian filter eliminate most of the noise and the contrast enhancement improve the visibility of our images and make the contours more pronounced and the value of PSNR became higher.

\section{B. Defect segmentation and location}

In this section, we present the results of the proposed segmentation method. Starting by image segmentation step which is proceeded as explained in Figure2.We applied
The chosen threshold level [33]. The optimal value we have calculated is 0.5 after that we applied adaptative thresholding command with MATLAB function (details of f) with this value and the result found is the input of the binarization.

In addition, we applied canny detector by fixing the values of Sensitivity threshold and the Standard deviation of the filter to 0.1 and 2 . We can detect the contours from binary image. Finally, we detect lines with Hough Transform with modification in the parameters. We specified optional parameters in the command of Hough peaks which indicate the numbers of peaks in Hough Matrix $\mathrm{H}$ to 8 and Threshold correspond to the minimum value to be considered a peak Ceil $\left(0.25^{*} \max (\mathrm{H}(:))\right)$ after that we extract line segments in binary image associated with particular bins in a Hough transform using the command MATLAB Houghlines with its input arguments theta and rho which are vectors returned by the Hough peaks that contains the row and column coordinates of the Hough transform bins to use in searching for line segments. Moreover, the others input arguments 'FillGap' and Minlength which is respectively the Distance between two lines segments associated with the same Hough transform bin equivalent to 120 and the Minimum line length equivalent to 20 .

The return value is a structure array whose length equals the number of merged line segments found. After that, we applied the procedure described in the section (C.2) to extract the line defect from the other detected lines. Results of segmentation are shown respectively in Fig.7 (a), (b), (c), and of location of linear defect by Hough Transform presented in Fig. 8.
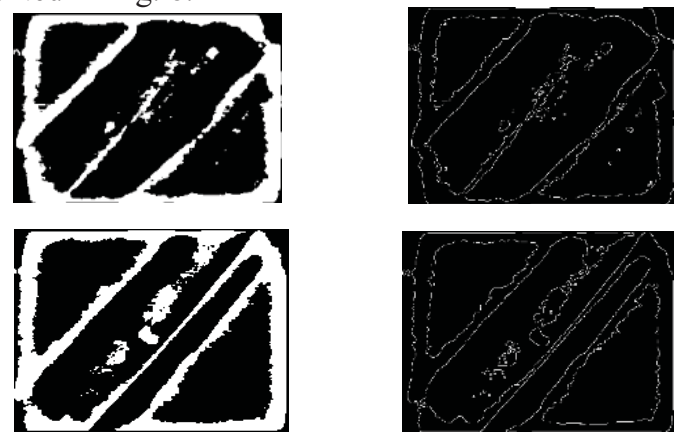

Fig. 7. Adaptive Thresholding, binarization and canny detector. (a) Applied to Fig (3.a); (b) Applied to Fig (3.b).

It can easily observe that applying the above segmentation steps our images are well segmented. Thus, we noted two classes of pixels in the image with the accentuation of defect's region, defect's zone is pronounced, and contours are outlined. PSNR value is calculated after process of segmentation and preprocessing image. We find that is equal to $51.7289 \mathrm{db}$. Finally, we applied Hough Transform to detect our defect's line, but we extract other lines, so we implemented an algorithm (C.2) to extract the defect's line by using the parameter of Hough $\rho$. The defect is always in the middle and is surrounded by two external lines shown in Fig8, so our goal is to detect and to draw the external lines and after that select the interior line which is the defect.

Indeed, we change the parameters of Hough to eliminate the lines borders by eliminating the angles $0^{\circ}$ and $90^{\circ}$ after than we applied the procedure explicated in section C.2. 
The resultant straight lines are shown in Fig. 8 plotted over the Canny detection image

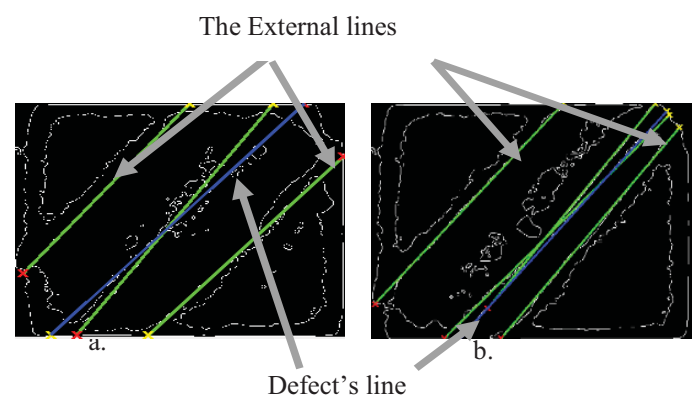

Fig. 8. Detected lines and defect's line on canny image, (a) Applied to Fig (3.a); (b) Applied to Fig (3.b).

We can deduce that one important difference between the Hough Transform and other traditional approaches like the edge detection methods (Laplacian,...) or applied in my previous article[32] is resistance of the former to noise in the image and its tolerance towards holes in the boundary line.

\section{$\mathrm{V}$. CONCLUSION}

This paper is an approach for automatic weld defect detection and localization from radiographic images. The proposed approach starts with image enhancement through contrast enhancement and noise reduction. After that the segmentation process of defects which is done by a combination of Adaptative Thresholding and Hough transform. So, we have performed at first Adaptative Thresholding and canny detector to segment the image and extract edges then Hough Transform to detect and locate the linear defect. The experimental results show that the proposed approach is reliable for the automatic defect detection from radiographic images in noisy environment

\section{REFERENCES}

[1] N. Nacereddine, M. Zelmat, S. S. Belaïfa and M. Tridi, "Weld defect detection in industrial radiography based digital image processing," Proceedings of world Academy of science Engineering and technology vol. 2, January 2005.

[2] M. Sezgin, B. Sankur, "Survey over image thresholding techniques and quantitative performance evaluation". Journal of Electronic imaging 13(1), Jan. 2004, pp. 146-165.

[3] J. Hassan, A. Majid Awan, A. Jalil, "Welding Defect Detection and Classification Using Geometric Features", 10th International Conference on Frontiers of Information Technology, 2012

[4] D Mery, M A Berti, "Automatic detection of welding defects using texture features”, (CTIP-2003), June 23-25, 2003, Berlin.

[5] Yan Wang, Yi Sun, Peng Lv, Hao Wang, "Detection of line weld defects based on multiple thresholds and support vector machine", NDT\&E International 41 (2008) 514-524

[6] Yan Wang, Yi Sun, Peng Lv, Hao Wang. Detection of line weld Defects based on multiple thresholds and support vectormachine. NDT\&E International 41 (2008) 514-524

[7] N. Nacereddine, M. Tridi, "Computer-aided Shape Analysis and Classification of Welded Defects in industrial Radiography based Invariant Attributes and Neural Networks", Proceeding of the 4th IEEE International Symposium on Image and Signal Processing and Analysis, Zagreb, Croatia, pp. 88-93, 2005.

[8] Rafael Vilar, Juan Zapata, Ramon Ruiz, "An automatic system of classification of weld defects in radiographic images," Ndt\&E International 42, 2009

[9] M. Sezgin, B. Sankur, "Survey over image thresholding techniques and quantitative performance evaluation," Journal of Electronic imaging 13(1), pp. 146-165, Jan. 2004.
[10] N. Nacereddine, L. Hamami, M. Tridi, and N. Oucief, "Non-parametric histogram-based thresholding methods for weld defect detection in Radiography," World Academy of Science, Engineering and Technology International Journal of Electrical, Electronic Science and Engineering, vol.1, 2007.

[11] G.T. Shrivakshan, Dr.C. Chandrasekar , "A Comparison of various Edge Detection Techniques used in Image Processing", IJCSI International Journal of Computer Science Issues, Vol. 9, Issue 5, No 1, September 2012

[12] H. Kälviäinen, P. Hirvonen, L. Xu and E. Oja, "Probabilistic and nonprobabilistic Hough Transforms: Overview and Comparisons," Image and Vision Computing, Vol. 13 N. 4, May 1995.

[13] Veer apathirapillai Vinoharan , Amirthalingam Ramanan, and Saluka R. Kodituwakku, "A Wheel-based Side-view Car Detection using Snake Algorithm”, ICIAfS'12 Radiographic image sequence",

NDT \& E International, Volume 46, March 2012, Pages 14-21

[14] N. Nacereddine, M. Zelmat, S. S. Belaïfa and M. Tridi, "Weld defect detection in industrial radiography based digital image processing," Proceedings of world Academy of science Engineering and technology vol. 2, January 2005.

[15] R. C. Gonzalez and R. E. Woods. Digital Image Processing. PrenticeHall, Inc., 2002.

[16] A. Kehoe, "The detection and evaluation of defects in industrial images," Ph.D. Thesis, University of Surrey, 1990.

[17] Y. Zheng, J.P. Basart, "Image analysis, feature extraction and various applied enhancement methods for NDE X-Ray images," Review of Progress in QNDE, vol. 7, pp. 813-820, 1988.

[18] Martine Bergounioux, "Quelques méthodes de filtrage en Traitement d'Image, " HAL archives-ouvertes, 29 Aug 2010 (v1).

[19] G. Deng and L. W, "An Adaptive Gaussian Filter For Noise Reduction and Edge Detection," Cahill.Nuclear Science Symposium and Medical Imaging Conference, 1993 IEEE Conference Record.

[20] S.S. Al-amri1, N.V. Kalyankar and. K.S.D, "Image Segmentation by Using Edge Detection,” Proc.(IJCSE) International Journal on Computer Science and Engineering, vol. 2, pp. 804-807, 2010.

[21] Antonio Alves de Carvalho, Raphael Carlos de Sá Brito Suita, Romeu Ricardo da Silva, João Marcos, and Alcoforado Rebello,"Evaluation of the Relevant Features of Welding Defects in Radiographic Inspection, "Materials Research, vol. 6, pp. 427-432, 2003.

[22] Xiangyang Xua, Yuan Zhoua, "Ultrasound intima-media segmentation using Hough transform and dual snake model," Computerized Medical Imaging and Graphics 36 (2012) 248-258 .

[23] Lucas Molina, Elyson A. N. Carvalho, "A Robotic Vision System Using a Modified Hough Transform to Perform Weld Line Detection on Storage Tanks," Robotic Symposium, 2008. LARS '08.

[24] Duda RO, Hart PE. Use of the Hough transformation to detect lines and curves in pictures. Communications of the ACM 1972.

[25] T. W. Liao and J. Ni, "An automated radiographic NDT system for weld inspection, Ch. I: Weld extraction,” NDT \& E Int. 29 (3), 157-162 (1996).

[26] G. A. Wang and T. W. Liao, "Automatic identification of different types of welding defects in radiographic images," NDT \& E Int. 35 (8), 519528 (2002).

[27] Noboyuki Otsu, A threshold selection method from gray level histograms IEEE transactions on systems, man, and cybernetics, 1979, Vol. SMC-9, No. 1: p. 62-66.

[28] Kass M, Witkin A, Terzopoulos D. Snakes,'active contour models, 'International Journal of Computer Vision 1988;1(4):321-31.

[29] Y. Zhang, and R. Webber, "A Windowing Approach to Detecting Line Segments Using Hough Transform", Pattern Recognition, Vol. 29, No. 2, 1996, pp. 255-265.

[30] Dong Jung Kang, Jang Won Choi, In So Kweon," Finding and Tracking Road Lanes using "Line-Snakes", semantic scholar, 2004.

[31] Gang Wang, T. Warren Liao,"Automatic identification of different types of welding defects in radiographic images," NDT\&E International 35(2002)519-520.

[32] ChirazAjmi, SabraElferchichi, KaotherLaabidi."New procedure for weld defect detection based Gabor filter," 2018 International Conference on Advanced Systems and Electric Technologies (IC ASET).

[33] N. Nacereddine, L. Hamami, M. Tridi, and N. Oucief," Non-Parametric Histogram-Based Thresholding Methods for Weld Defect Detection in Radiography", International Journal of Electrical, Electronic Science and Engineering Vol:1 No:9, 2007. 